\title{
Producción de pulpa de fruta de cerezo (Malpghia punicifolia l.) y su potencial nutricional para consumo humano
}

Production of cherry fruit pulp (Malpghia punicifolia L.) and its nutritional potential for human consumption

Sandra Milena Daza Orsini

Magister en Ciencia y Tecnología de alimentos, Universidad de la Guajira.

Correo electrónico: smdaza@uniguajira.edu.co

\section{Oscar José Lindo Oñate}

Especialista en Gerencia Social, Universidad de la Guajira.

Correo electrónico: oscarlindo@uniguajira.edu.co 


\section{Resumen}

El fruto del cerezo (Malpghia punicifolia L.), es comercializado por la población Wayuu, el cual se conoce como cereza, es muy perecedero; lo que origina perdidas en épocas de cosecha. Debido a esto, se vio la necesidad de efectuar una investigación que tiene por objetivo realizar un proceso tecnológico para la producción de pulpa de fruta y conocer las características nutricionales y microbiológicas para garantizar la inocuidad del producto terminado. En los resultados se identificó que el estado ideal de maduración para el procesamiento del fruto cerezo es el grado 5, reconocido con el color rojo. En la pulpa de fruta del cerezo se determinó que obtuvo un $\mathrm{pH}$ de 3.8, unos grados ${ }^{\circ}$ Brix de 12.89 y una acidez de $2.11 \%$ expresada en ácido cítrico, $1.33 \%$ de proteína y minerales como hierro, calcio, potasio y fosforo, además está libre de microorganismos patógenos como la E.coli y salmoneslla. Se considera con este estudio que es una alternativa de procesar frutos nativos de la región a través de la producción pulpa de frutas ya que es un producto alimento con alto valor nutricional y lo hace atractivo para su posterior uso agroindustrial ya sea en néctares, mermeladas, yogures o conservas.

Palabras clave: valor nutricional, producto alimenticio, uso agroindustrial.

\section{Abstract}

The fruit of the cherry tree (Malpghia punicifolia L.), is commercialized by the Wayuu population, which is known as cherry, is very perishable which causes losses during harvest, due to this it was necessary to carry out an investigation where it has as an objective to carry out a technological process for the production of fruit pulp and to know the nutritional and microbiological characteristics to guarantee the safety of the finished product. In the results it was identified that the ideal state of maturation for the processing of the cherry fruit is grade 5, recognized with the color red. In the pulp of fruit of the cherry tree it was determined that it obtained a $\mathrm{pH}$ of 3.8 , some degrees ${ }^{\circ}$ Brix of 12.89 and an acidity of $2.11 \%$ expressed in citric acid, $1.33 \%$ of protein and minerals such as iron, calcium, potassium and phosphorus. free of pathogenic microorganisms such as E. coli and salmonella. It is considered with this study that it is an alternative to process native fruits of the region through the production of fruit pulp, since it is a food product with high nutritional value and makes it attractive for its later agro-industrial use, whether in nectars, jams, yoghurts or preserves.

Keywords: nutritional value, food product, agro-industrial use.

\section{Introducción}

El cerezo (cuyo nombre guajiro es Jai' pai, como se le reconoce por la población indígena Wayuu), es un fruto que pertenece a la familia Malpighiaceae y su nombre científico es Malpighia puniciflora L., es una fruta silvestre que se da en la zona norte de Colombia, tiene propiedades nutricionales que la hacen atractiva para elaborar productos industriales para consumo humano que pueden llegar a satisfacer necesidades alimenticias. Dentro de la clasificación, según la parte utilizada de la planta, es un fruto; además, pertenece a la categoría de frutas tropicales debido a que se cultiva en climas tropicales y subtropicales. La cosecha de este fruto se da en los meses de mayo, septiembre y octubre, principalmente en la zona de la media y alta Guajira.

El cerezo (Jai'Pai) es una especie arbustiva de mucho valor para los indígenas Wayuu. El árbol mide entre 3 a $4 \mathrm{~m}$ de altura, posee un tallo leñoso de color marrón blancuzco; sus raíces son delgadas y quebradizas. Las hojas son opuestas, lanceoladas, pinnatinervias de color verde claro, con el ápice obtuso y la base aguda. La inflorescencia presenta de 3-6 flores con 5 pétalos lilas, el fruto 
es globuloso de color rojo, de sabor agridulce (Rosado, 2009).

Este fruto tiene tres semillas que representan entre el 19 y el $25 \%$ del peso total, su tamaño varía entre de 1 a $2,5 \mathrm{~cm}$, el diámetro de 1 a $4 \mathrm{~cm}$ y el peso de 2 a 15 g. Presenta una coloración verde cuando está en desarrollo, cambiando a tonos amarillos y rojos cuando está maduro (Alves \& Menezes, 1995).

Sin embargo, este fruto en el Departamento de la Guajira no se aprovecha industrialmente, por lo tanto, se hace atractivo para implementar procesos agroindustriales, los cuales generarían una diversidad de productos procesados permitiendo alargar la vida útil del fruto debido a que es altamente perecedero. Entre esta diversidad de productos se encuentra la producción de pulpa de fruta, el cual es el objeto de estudio de este trabajo. La idea es implementar un esquema tecnológico para estandarizar un diagrama de proceso y, asimismo, caracterizar bromatológicamente la pulpa de fruta con el fin de conocer las propiedades nutricionales de este producto.

\section{Materiales o recursos}

Se emplearon frutos de cerezo (Malpghia punicifolia L.), adquiridos en el mercado público de la ciudad de Riohacha. Luego, se procedió con la parte experimental en las instalaciones de la Planta Piloto de Procesamiento Pesquero de la Universidad del Magdalena, ubicada Cr 2 No. 18-27 del corregimiento de Taganga, distrito de Santa Marta.

\section{Equipos}

La producción de la pulpa de fruta a partir del fruto de cerezo (Malpghia punicifolia L.), se obtiene a través de unos equipos especiales, que se usan durante las etapas de proceso y en la realización de análisis (indispensables para garantizar la inocui- dad de la pulpa de fruta). Entre los equipos utilizados se encuentran:

- Potenciómetro

- Refractómetro

- Despulpadora

- Selladora de bolsas

- Tanque escaldador

- Balanza analítica

- Mesas en acero inoxidables

- Intercambiador de calor de tubo

- Congelador

\section{Elaboración y estandarización de la pulpa de fruta}

En la presente investigación se analizó y describió cada una de las técnicas utilizadas en el proceso de obtención de la pulpa de fruta a partir del cerezo (Malpighia puniciflora L.). El procedimiento consistió en seleccionar varias muestras representativas del fruto y diseñar un diagrama de proceso, donde, se describen las operaciones indicadas para obtener como producto final la pulpa de fruta, el cual esta descrito en la figura 1.

\section{Descripción del diagrama de proceso para la elaboración de la pulpa de fruta}

Recepción de materia prima: los frutos del cerezo fueron adquiridos en el mercado púbico de la ciudad de Riohacha, departamento de la Guajira, Colombia.

Pesado 1: los frutos enteros fueron pesados en una balanza analítica de precisión, con el fin de determinar el rendimiento del proceso.

Selección y/o clasificación: en esta etapa se selecciona la fruta sana de la que no es apta para el procesamiento de la pulpa de fruta, como aquellas que presentan magulladuras, golpes, rajaduras, hongos, laceraciones, picaduras de insectos 
Lavado y desinfección: con agua clorada (2 ppm) buscando reducir la carga bacteriana y eliminar impurezas y suciedades.

Pesado 2: se determinó el peso de las frutas que cumplieran con los parámetros de selección establecidos para obtener el rendimiento del producto final.

Escaldado: El fruto del cerezo se coloca en un tanque de escaldado a una temperatura de $75^{\circ} \mathrm{C}$ durante cinco minutos, el objetivo de este proceso es ablandar la fruta previa para la etapa del despulpado, inactivar enzimas y reducir la carga microbiana presente en el fruto.

Despulpado: los frutos del cerezo se introducen en una despulpadora, con el fin de separar la semilla de la cascara y obtener la pulpa de fruta.

Pasteurizado: la pulpa del fruto del cerezo es llevada a un intercambiado del calor por una temperatura de $90^{\circ} \mathrm{C}$ durante 3 minutos, para su posterior envasado y sellado.

Envasado y sellado: la pulpa de fruta de cerezo se empaca en bolsas de polietileno de polietileno de alta densidad y sellado de manera hermética en unidades de $250 \mathrm{ml}$. El sellado se realiza a través de una selladora eléctrica.

Almacenamiento: la pulpa de fruta se almacenó en un congelador a una temperatura $0^{\circ} \mathrm{C}$ a -5 , con el fin de aumentar la vida útil del producto que es de 6 a 12 meses.

\section{Caracterización bromatológica}

Posteriormente se realiza la caracterización bromatológica de la pulpa de fruta con el objetivo de determinar su valor nutricional, el cual se efectúa mediante las técnicas del análisis proximal según AOAC (1990) (como se puede observar en la tabla 1).

\begin{tabular}{|l|l|}
\hline Determinación & Técnica analítica utilizada \\
\hline Humedad & Método gravimétrico \\
\hline Cenizas & Método gravimétrico \\
\hline Proteínas & Método de kjeldahl \\
\hline Grasas & $\begin{array}{l}\text { Método gravimétrico (Método } \\
\text { Howard) }\end{array}$ \\
\hline Solidos solubles & Refractómetro \\
\hline hierro, calcio, potasio, & Método de absorción atómica \\
\hline Fosforo & Colorimétrico con ácido ascórbico \\
\hline Acidez & $\begin{array}{l}\text { Método A.O.A.C. } \\
11.042 / 84.962 .12 / 90\end{array}$ \\
\hline pH & 11.043/84.964.08/90(1990) \\
\hline
\end{tabular}

Tabla 1. Métodos utilizados para la obtención de análisis bromatológico. 


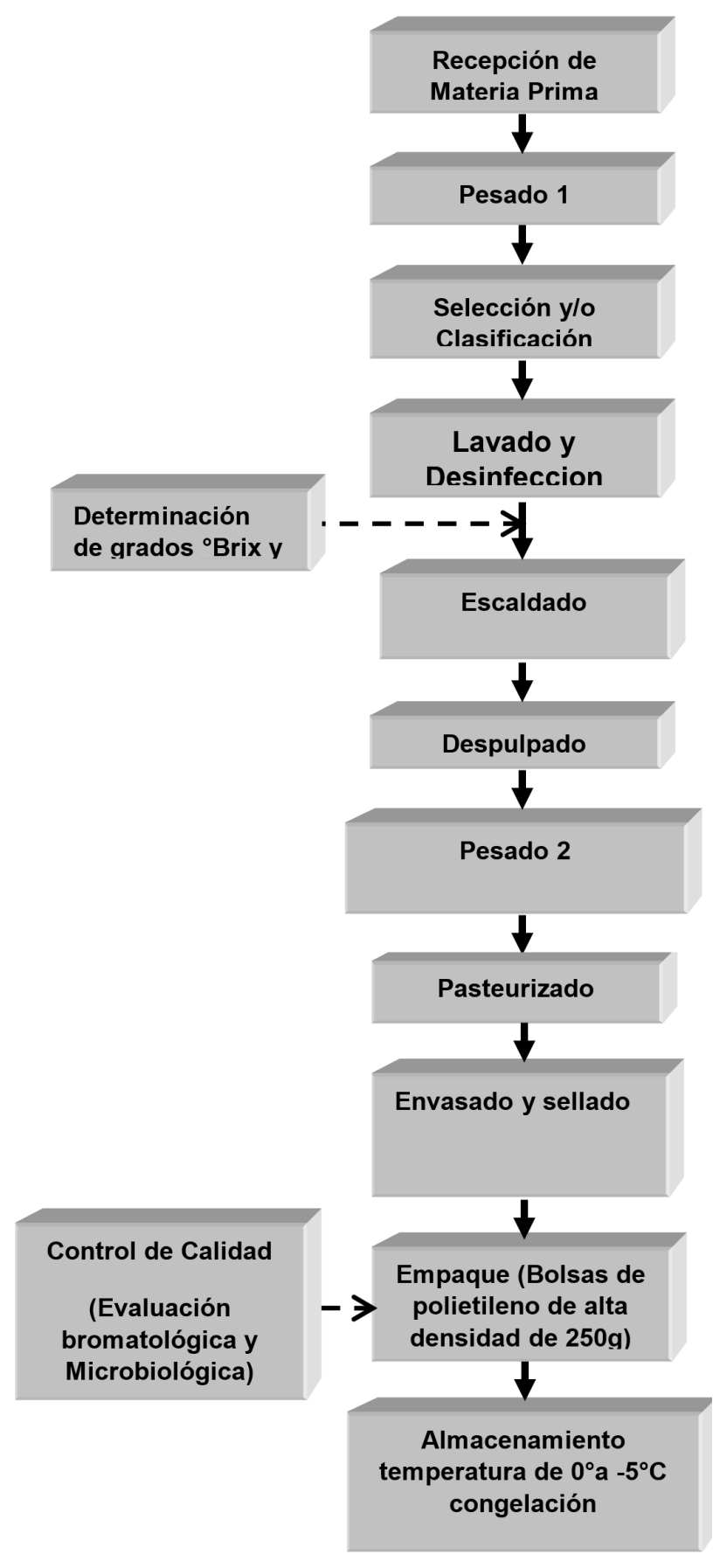

Figura 1. Diagrama de flujo de la producción de la pulpa de fruta a partir del cerezo (Malpighia puniciflora L.)

\section{Pruebas microbiológicas del producto terminado}

La pulpa de fruta a partir del fruto del cerezo (Malpighia puniciflora L.) se le realiza un análisis microbiológico para determinar sì existe E. Coli, salmonella, hongos, levaduras y garantizar que sea un producto inocuo, libre de microorganismos patógenos, esto se hace según la resolución 3929 del 2013.

\section{Resultados y discusión}

\section{Análisis realizados a la fruta del cerezo (Malpighia puniciflora L.) para determinar el grado de madurez óptimo para la elaboración de pulpa de fruta.}

Se establecieron cinco grados de color de acuerdo con la evolución de la maduración del fruto del cerezo y las necesidades del mercado y de los consumidores (ver figura 2). En la tabla 2 se describen los grados de maduración de acuerdo al color del fruto, donde el grado 1 es la pertenece a la coloración inmadura y el grado 5 corresponde al grado óptimo de maduración para elaborar pulpas de frutas; además se observan los grados ${ }^{\circ}$ Brix, solidos solubles ideales para el procesamiento del fruto, donde indica que entre mayor sean, corresponde a la maduración y el menor, el fruto no ha alcanzado su madurez. 


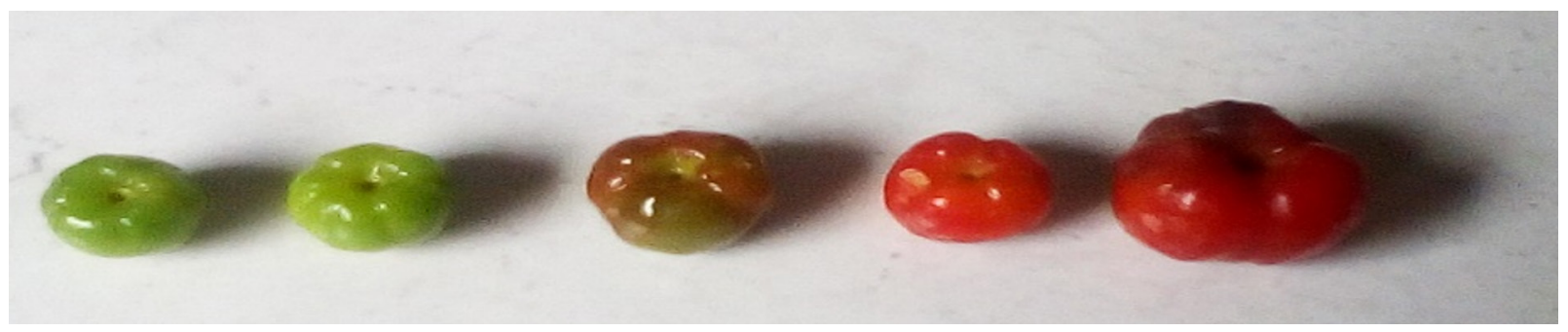

Figura 2. Estados de maduración del fruto del cerezo (Malpighia puniciflora L.)

\begin{tabular}{|l|l|l|}
\hline Grado de Maduración & Características & ${ }^{\circ}$ Brix (\%) \\
\hline Grado 1. Verde lima & $\begin{array}{l}\text { Color normal de la fruta fresca. En un estado de madurez inapropiado para la elaboración de la } \\
\text { pulpa de fruta. }\end{array}$ & 5 \\
\hline $\begin{array}{l}\text { Grado 2. Ligeramente } \\
\text { verde }\end{array}$ & Primer cambio de color durante el ciclo de maduración. & 8 \\
\hline Grado 3. Verde rojizo & Recomendado para la distribución ya que su proceso de maduración es acelerado. \\
\hline $\begin{array}{l}\text { Grado 4. Ligeramente } \\
\text { rojo }\end{array}$ & Pronunciado estado inicial del ciclo de maduración, ideal para consumirlo fresco y realizar jugos. & 12 \\
\hline Grado 5 Rojo & $\begin{array}{l}\text { Ideal para el consumo y procesamiento de productos (bocadillos, mermelada, jugos, pulpas } \\
\text { de frutas). Color rojo con un alto valor nutritivo y procesamiento rápido por su alto estado de } \\
\text { maduración. }\end{array}$ & 12.89 \\
\hline
\end{tabular}

Tabla 2. Métodos utilizados para la obtención de análisis bromatológico.

Como se observa, durante la maduración del fruto del cerezo se presenta un aumento de los sólidos solubles ( ${ }^{\circ}$ Brix, una variable que indica la cantidad de azúcar presente), es por eso que el fruto entre más maduro, obtiene un coeficiente mayor de grados ${ }^{\circ}$ Brix, es más dulce; en un grado de madurez inmaduro se obtuvo un valor muy bajo, el cual fue de $5^{\circ}$ Brix, este indica que no es ideal para el procesamiento del fruto en pulpa de fruta.

\section{Análisis realizados a la pulpa de fruta producida a partir del fruto del cerezo (Malpighia puniciflora L.)}

\section{Análisis bromatológico}

En la tabla 3, se observa el resultado del análisis bromatológico que se realizó a la pulpa de fruta después del procesamiento del fruto del cerezo, con el objetivo de determinar los componentes alimenticios presente en este producto, como: carbohidratos, fibra, proteína, cenizas, humedad y minerales presentes como calcio, potasio, hierro y fosforo. 


\begin{tabular}{|l|l|}
\hline Parámetro & Pulpa fruta \\
\hline Humedad \% & 81,23 \\
\hline Proteína \% & 1,33 \\
\hline Grasas \% & 0,34 \\
\hline Carbohidrato \% & 14,21 \\
\hline Fibra cruda \% & 1,88 \\
\hline Cenizas \% & 0,98 \\
\hline Potasio mg & 211 \\
\hline Fosforo mg & 17,67 \\
\hline Calcio mg & 15,4 \\
\hline Hierro mg & 0,44 \\
\hline *Sólidos solubles (Brix ${ }^{\circ}$ ) & 12,89 \\
\hline *pH & 3.8 \\
\hline *Acidez titulable\% & 2,11 \\
\hline
\end{tabular}

Tabla 3. Contenido bromatológico de la pulpa de 1 fruta del cerezo (Malpighia puniciflora L.)

Nota: Los parámetros que contiene $\left(^{*}\right)$ pertenecen a las características fisicoquímicas de la pulpa de fruta.

Se puede afirmar que la pulpa de fruta a partir del cerezo, presenta características fisicoquímicas ideales para el procesamiento de este fruto, permitiendo indagar algunas posibilidades de agroindustrializacion, ya que presenta un $\mathrm{pH}$ bajo que es de 3.8, característica ideal para que no desarrollen microorganismos en medio ácido y también lo hace atractivo para elaborar mermeladas, néctares y bocadillo. En cuanto al porcentaje de acidez titulable, arrojó un resultado del 2.11\% (el ácido presente en la pulpa de fruta es el ácido cítrico). El porcentaje de humedad de la pulpa de fruta es de $81.23 \%$, lo cual indica que se debe usar un método de conservación ideal que no afecte sus características organolépticas y nutricionales, en este caso se optó por usar la congelación con una temperatura de almacenamiento entre 0 y $-5^{\circ} \mathrm{C}$. Por otra parte, se destaca el nivel proteico de la pulpa de fruta a partir del fruto del cerezo, ya que por ser origen vegetal presenta un $1.33 \%$ de proteína (considerado benéfico para la salud del consumidor). Además, en la pulpa de fruta se encontraron minerales como el hierro, calcio fósforo y potasio; el que este producto presente hierro lo hace atractivo para aquellas personas que tienen deficiencia de este mineral en su organismo, así el consumo de la pulpa de fruta del cerezo, contrarrestaría los niveles de anemia que se encuentran en la población Guajira.

\section{Análisis microbiológicos}

Los resultados señalaron que el número más probable de E. coli en la muestra de la pulpa de la fruta del cerezo (Malpighia puniciflora $L$ ) fue igual a cero y el recuento de hongos y levaduras fue $<100$; además fue negativa la presencia de salmonella, como se puede observar en la tabla 4 . Todos estos resultados garantizan la inocuidad de la pulpa de fruta, obteniendo garantías para el consumo de este producto.

\begin{tabular}{|l|l|l|}
\hline Parámetros & $\begin{array}{l}\text { Muestra pulpa } \\
\text { de fruta }\end{array}$ & $\begin{array}{l}\text { Ref. Resolución } \\
3929 / 2013\end{array}$ \\
\hline Recuento de E. coli UFC/g & 0 & $<10$ \\
\hline $\begin{array}{l}\text { Recuento de hongos y } \\
\text { levaduras UFC/g }\end{array}$ & 18 & $<100$ \\
\hline Salmonella/25g & Ausente & Ausente \\
\hline
\end{tabular}

Tabla 4. Microorganismos patógenos presentes en la muestra de pulpa de fruta del fruto del cerezo (Malpighia puniciflora $L$ )

\section{Conclusiones}

El estado de maduración e adecuado para la fabricación de la pulpa de fruta fue grado 5 , en este estado el fruto presenta textura firme y ligeramente blanda, favorable para la manipulación de la fruta al momento de realizar la operación de despulpado.

Los resultaron arrojaron que los primeros estados de madurez del fruto es grado 1 y 2 , el contenido de azúcar es mínimo presentando unos ${ }^{\circ}$ Brix de 5 y 8 respectivamente, luego a partir del grado 3 y 
al ir avanzando la maduración, tienden a aumentar los grados ${ }^{\circ}$ Brix con un valor de 12.89 , correspondiente al estado de madures de grado 5 . A nivel industrial, sí se quiere obtener un producto con una concentración de azúcar este sería el estado óptimo de maduración del fruto de cerezo.

El porcentaje de grasa en la pulpa de fruta del cerezo fue de $0.34 \%$, es un valor muy bajo, el cual lo hace atractivo para aquellas personas que están en proceso alimentarse sanamente (presenta un porcentaje de proteína de $1.33 \%$ ); además es rico en fosforo, calcio, potasio y hierro.

El contenido de fibra encontrado para la pupa de fruta del fruto del cerezo (Malpighia puniciflora L.) fue igual a $1.88 \%$, carbohidratos totales $14.81 \%$ siendo este el mayor componente alimenticio que tiene el producto y que constituye una fuente de energía.

En la pulpa de fruta del fruto del cerezo (Malpighia puniciflora L.) se constató que estuviera libre de contaminación debido a que no existe la presencia de E. Coli y salmonella, microorganismos patógenos que afectan la salud del consumidor.

El cerezo (Malpighia puniciflora L.), es un fruto con perspectivas de agroindustralización, pues se pueden obtener una variedad de productos como mermeladas, compotas y néctares debido a los parámetros fisicoquímicos obtenidos en la investigación.

La pulpa de fruta del cerezo (Malpighia puniciflora L.), es un producto innovador, con una vida útil de seis a doce meses con una temperatura de almacenamiento entre $0 \mathrm{a}-5^{\circ} \mathrm{C}$ y además posee excelentes propiedades nutricionales, por lo tanto, se recomienda realizar un plan de marketing para dar a conocer el producto y así lograr una exitosa distribución en el mercado local, regional y nacional.

\section{Referencias}

AOAC. (1990). Official Methods of Analysis. Washington DC: Association of Official Analytical Chemists.

Alves, R. E. \& Menezes, J.B. (1995). Botânica da aceloreira. En A. São José \& R. E. Alves (Ed.), Acerola no Brasil: produção e mercado (pp. 7-14). Salvador de Bahía: Universidade Estadual do Sudoeste da Bahia.

Camacho, G. (2004). Obtención y conservación de pulpas de frutas. Bogotá: Universidad Nacional de Colombia.

Desrosier, N.W. (1980). Conservación de alimentos. México: Editorial Continental.

Fenema, O. (1993). La ciencia de los alimentos. Zaragoza: Editorial Acribia.

Meyer, M. \& Paltrinieri, G. (1993). Manual de elaboración de frutas y hortalizas. Ciudad de México: Editorial Trillas.

Rosado, J. (2009). Farmacopea guajira, cosmovisión y usos de las plantas medicinales por los Wayuu. Riohacha: Universidad de la Guajira. 